\title{
Relationship of Average Transmitted and Received Energies in Adaptive Transmission
}

\author{
Aarne Mämmelä, Senior Member, IEEE, Adrian Kotelba, Member, IEEE, Marko Höyhtyä, Student Member, IEEE, \\ and Desmond P. Taylor, Life Fellow, IEEE
}

\begin{abstract}
This paper studies the analytical relationship between the average transmitted and received energies under several adaptive transmitter power control methods, including water filling, truncated power inversion, and downlink beamforming. The study is applicable to many fading channel scenarios, including frequency-nonselective, frequency-selective, and multiple-inputmultiple-output (MIMO) channels. Both the average transmitted and received energies are commonly used in performance comparisons, and the selection depends on what one wants to investigate. The transmitted energy is known to be the basic system resource. In the case of adaptive transmission, the average transmitted energy should, in general, be used instead of the average received energy. The use of transmitted energy leads to the normalization problem of the channel. The ratio of received energy to transmitted energy is the energy gain of the channel. All physical systems follow an energy-conservation law, which implies that the energy gain of the channel is less than or equal to 1 . The major approaches for normalization include the setting of either the average energy gain or the peak energy gain to unity. In the normalization, the average energy gain is defined for a signal whose energy is uniformly distributed across the frequency and spatial dimensions. The peak energy gain of many mathematical fading models is not bounded, and those models cannot be normalized by the peak energy gain. We show that the proper normalization of the mathematical model and the selection of the correct performance measure are of critical importance in comparative performance analysis of adaptive transmission systems.
\end{abstract}

Index Terms-Energy-conservation law, multiantenna systems, multipath fading, multiple-input-multiple-output (MIMO) systems, transmitter power control.

\section{INTRODUCTION}

$\mathbf{E}$ NERGY is a basic resource in digital transmission links, and systems should be analyzed on the basis of how efficiently it is used [1]. We divide systems into energy limited and power limited. In power-limited systems, such as a base station connected to the electrical network, the available power is limited, but energy is essentially unbounded. In energylimited systems, such as a mobile terminal using a battery, the available energy is limited. In practice, to make the problem

Manuscript received June 30, 2009; revised November 5, 2009. First published December 28, 2009; current version published March 19, 2010. This work was supported in part by the Scientific Advisory Board for Defence (Matine). This paper was presented in part at the 2005 IEEE Global Telecommunications Conference (GLOBECOM'05), GLOBECOM'06, and GLOBECOM'07. The review of this paper was coordinated by Prof. H. H. Nguyen.

A. Mämmelä, A. Kotelba, and M. Höyhtyä are with VTT Technical Research Centre of Finland, 90571 Oulu, Finland (e-mail: aarne.mammela@vtt.fi; adrian.kotelba@vtt.fi; marko.hoyhtya@vtt.fi).

D. P. Taylor is with the University of Canterbury, Christchurch 8140, New Zealand (e-mail: taylor@elec.canterbury.ac.nz).

Digital Object Identifier 10.1109/TVT.2009.2039156 analytically tractable, systems are usually optimized as if they were power limited, but performance is often measured as if they were energy limited by using the energy-to-noise power spectral density ratio.

There appear to be two alternative modes of performance measurement in terms of energy. Either the average transmitted or received energy per symbol is used, and both are usually normalized by the receiver noise spectral density. This leads to the average transmitted SNR per symbol [2, pp. 40-127], [3][6] and average received SNR per symbol [7], [8], respectively. The use of average transmitted and received SNRs was briefly discussed in [9, p. 2628], but no recommendation was made concerning their preferred usage.

It is known that system performance depends in part on the average received SNR. If we compare different receivers for the same transmitted signal and for the same channel, we can measure how well the receiver is matched to the channel. However, we do not necessarily know how well the transmitted signal is matched to the channel. In energy-limited systems, the transmitted energy is the basic system resource since it is the transmitted energy rather than the received energy that is taken from the battery and is necessarily limited. The actual received energy is typically a small fraction of the transmitted energy due to the finite effective aperture of the antennas. The authors of [7] and [8] optimize the use of the transmitted energy in adaptive transmission in a fading channel, but their numerical results show how efficiently the received energy is used. The reader is led to believe that the system is almost as good as if there were no fading at all, although significant improvement is actually possible. The use of the received SNR was emphasized in [10], but when the transmitter was optimized [10, p. 572], the transmitted power was fixed, following the theory presented in [2].

Since the average energy gain of the channel is a function of the transmitted signal, particularly in adaptive transmission systems, it is crucial to use the transmitted SNR rather than the received SNR for performance measurements. However, it is still possible to use the average transmitted SNR referred to the receiver, which is to be defined in Section III-E, provided that the channels are properly normalized. The average received SNR can be used if the channels are correctly normalized and the transmitters exploit no form of channel selectivity.

The major approaches for normalization of the channel include normalization of the average energy gain or the peak energy gain to unity [11]. For brevity, we call them average and peak normalization, respectively. In the normalization, the average energy gain is defined for a signal whose energy is uniformly distributed across the frequency and spatial dimensions. 
We will refer to this specific average energy gain as the representative energy gain. Energy conservation is known to hold for all physical systems. Therefore, the output energy of a passive system cannot be larger than the input energy, and the peak energy gain should be less than or equal to 1 because a major part of the energy is lost in the channel from the receiver point of view. In most of the literature on fading channels, average normalization is used. However, as is shown in [11] and in the present paper, this approach must be reconsidered when either the transmitted signal or the channel exhibits selectivity in time, frequency, or space. The authors of [11] noticed that the peak energy gain of a linear time-invariant frequency-selective filter can be larger than unity if average normalization is used.

Our major contribution is to extend the results of [11] to a general class of linear vector channels. A linear vector model can represent a wide range of physical channels. These vector channels can be time variant or time invariant, frequency selective or frequency nonselective, and may have many inputs or outputs corresponding to, for example, multiple antennas. These systems are usually called multiple-input-multipleoutput (MIMO) systems. Finally, the transmitter may use an arbitrary power control scheme. We show the analytical relationship between the average transmitted and received SNRs by using the covariance between the transmitted energy and the energy gain of the channel. The covariance specifies how well the transmitted signal is statistically matched to the channel. We also show that the conclusions from performance comparisons depend on whether the transmitted or the received SNR is used in the comparisons. In general, the transmitted SNR should be used, and the proper normalization method is shown to be peak normalization. We present novel bounds on the average received SNR that can be achieved with an adaptive powercontrol scheme and generalize previous analysis [11] to include time, frequency, and spatial domains.

Parts of this paper were presented in our earlier conference papers [12]-[14]. In the present combined paper, we have extended these results and have unified and elaborated the explanations and examples. We have made a clear distinction between power- and energy-limited systems. We have also derived analytical expressions for the distributions of the SNRs and shown the deviations if the channel is not peak normalized.

The remainder of this paper is organized as follows: In Section II, we introduce models of a linear vector channel and an adaptive transmitter. The basic concepts related to the transmission of energy through linear vector channels are covered in Section III. Section IV contains analysis for frequencyselective and frequency-nonselective fading channels with multiple antennas. Numerical results are presented in Section V and conclusions in Section VI.

Notation: Boldface lowercase letters $\boldsymbol{a}$ denote column vectors; $\boldsymbol{A}=\left[a_{i j}\right]_{i, j=1}^{m, n}$ denotes an $m \times n$ matrix whose $(i, j)$ th entry is $a_{i j} ;(\cdot)^{*}$ denotes the conjugate transpose of a matrix; $\operatorname{tr}(\cdot)$ is the trace of a matrix; $\operatorname{rank}(\cdot)$ is the rank of a matrix; $\operatorname{diag}\left(d_{11}, \ldots, d_{k k}\right)$ is a $k \times k$ diagonal matrix with entries $d_{i i}$, $1 \leq i \leq k ; \boldsymbol{I}_{m}$ is the $m \times m$ identity matrix; $\left\{a_{i}\right\}_{i=1}^{u}$ denotes an ordered set of $u$ elements, $a_{1} \leq a_{i} \leq a_{u}$; $\operatorname{Pr}[\cdot]$ denotes probability; $\mathbb{E}[X]$ and $\bar{X}$ denote expectation of a random variable $X ; \mathbb{E}[X \mid Y]$ denotes conditional expectation of a random variable $X$ given a random variable $Y$; $\operatorname{Var}[\cdot]$ denotes variance of a random variable; $\operatorname{Cov}[\cdot, \cdot]$ denotes the covariance of two random variables; $f(x)^{+}=\max [0, f(x)]$; and $\mathbb{C}$ and $\mathbb{R}$ denote the fields of complex and real numbers, respectively.

\section{SySTEM MODEL}

\section{A. Channel Model}

We consider a discrete-time linear vector channel with $n$ inputs and $m$ outputs, where $n \leq m$ for reliable detection. Let $\boldsymbol{y} \in \mathbb{C}^{n}$ denote a vector of complex input symbols, $\boldsymbol{n} \in \mathbb{C}^{m}$ denote a vector of complex noise samples, and $r \in \mathbb{C}^{m}$ be a vector of complex output symbols. The output $\boldsymbol{r}$ and input $\boldsymbol{y}$ symbols are related by the matrix equation [15]-[18]

$$
\boldsymbol{r}=\boldsymbol{H} \boldsymbol{y}+\boldsymbol{n}=\boldsymbol{z}+\boldsymbol{n}
$$

where $\boldsymbol{z}=\boldsymbol{H} \boldsymbol{y}$ denotes the signal component of the received signal. The complex channel coefficients between the $j$ th input and the $i$ th output, which is denoted by $h_{i j}$, are assembled into a channel matrix $\boldsymbol{H}=\left[h_{i j}\right]_{i, j=1}^{m, n}$. We assume that the entries of $\boldsymbol{n}$ are complex zero-mean Gaussian random variables with variance $\sigma_{n}^{2}=N_{0} / T_{s}$, where $N_{0}$ denotes the noise power spectral density, and $T_{s}$ is the sampling interval. Furthermore, we assume that the noise samples are uncorrelated, that is, $\mathbb{E}\left[\boldsymbol{n n}^{*}\right]=\sigma_{n}^{2} \boldsymbol{I}_{m}$.

The vector channel defined in (1) models a wide range of physical channels.

1) Frequency-nonselective fading channel with a single antenna- $\boldsymbol{H}$ is a random scalar.

2) Frequency-selective time-invariant channel with a single antenna- $\boldsymbol{H}$ is an arbitrary Toeplitz matrix that is fixed for the whole transmission duration.

3) Frequency-nonselective fading channel with multiple antennas $-\boldsymbol{H}$ is an arbitrary matrix that randomly changes from one channel use to another.

4) Frequency-selective block-fading channel with a single antenna- $\boldsymbol{H}$ is an arbitrary Toeplitz matrix that randomly changes from one transmission block to another.

5) Frequency-selective block-fading channel with multiple antennas $-\boldsymbol{H}$ is a block-Toeplitz matrix that randomly changes from one transmission block to another.

Frequency-selective fading can be modeled by (1), provided that the channel memory is assumed to be finite. The channel matrix $\boldsymbol{H}$ is then, as will be shown later, a convolution matrix with a Toeplitz or a block-Toeplitz structure.

The performance analysis is simplified if the channel model described by the matrix $\boldsymbol{H}$ is transformed into a virtual set of parallel orthogonal subchannels [15]-[18]. The transformation of the channel into its virtual structure is achieved with singular value decomposition. Let $\left\{\lambda_{i}\right\}_{i=1}^{u}$ be the nonzero eigenvalues of the matrix $\boldsymbol{A}=\boldsymbol{H}^{*} \boldsymbol{H}$. Then, we may write [19, p. 193]

$$
\boldsymbol{H}=\boldsymbol{U} \boldsymbol{D} \boldsymbol{V}^{*}
$$

where $\boldsymbol{U} \in \mathbb{C}^{m \times m}$ and $\boldsymbol{V} \in \mathbb{C}^{n \times n}$ are unitary, and the $m \times n$ diagonal matrix $\boldsymbol{D}$ has $\sqrt{\lambda_{i}}$ in the $(i, i)$ position $(1 \leq i \leq u)$ 


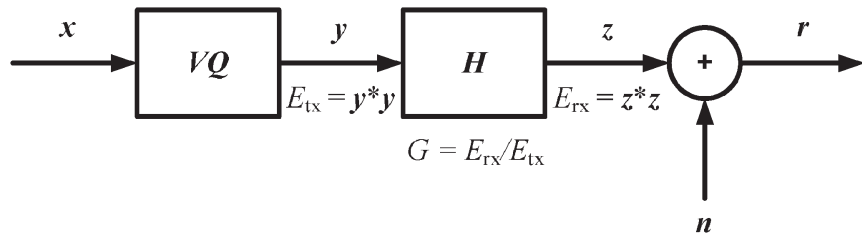

Fig. 1. Block diagram of the system.

and zeros elsewhere. The matrix equation (1) can then be rewritten as

$$
\boldsymbol{r}=\boldsymbol{U} \boldsymbol{D} \boldsymbol{V}^{*} \boldsymbol{y}+\boldsymbol{n} .
$$

Finally, the vector channel model is transformed into a set of orthogonal subchannels when the input and output vectors $y$ and $\boldsymbol{r}$ are left multiplied by matrices $\boldsymbol{V}^{*}$ and $\boldsymbol{U}^{*}$, respectively. Thus, we obtain

$$
\tilde{\boldsymbol{r}}=\boldsymbol{U}^{*} \boldsymbol{r}=\boldsymbol{D} \boldsymbol{V}^{*} \boldsymbol{y}+\boldsymbol{U}^{*} \boldsymbol{n}=\boldsymbol{D} \tilde{\boldsymbol{y}}+\tilde{\boldsymbol{n}}
$$

where $\tilde{\boldsymbol{y}}=\boldsymbol{V}^{*} \boldsymbol{y}$, and $\tilde{\boldsymbol{n}}=\boldsymbol{U}^{*} \boldsymbol{n}$.

The channel model described by $\boldsymbol{H}$ and its virtual representation as specified by the unitary matrices $U$ and $\boldsymbol{V}$ and the diagonal matrix $D$ are equivalent in the sense that the total input and output energies remain the same, i.e., $\boldsymbol{z}^{*} \boldsymbol{z}=\tilde{\boldsymbol{z}}^{*} \tilde{z}$, $\boldsymbol{n}^{*} \boldsymbol{n}=\tilde{\boldsymbol{n}}^{*} \tilde{\boldsymbol{n}}$, and $\boldsymbol{y}^{*} \boldsymbol{y}=\tilde{\boldsymbol{y}}^{*} \tilde{\boldsymbol{y}}$. This is due to the fact that the inner product is invariant to unitary similarity transformations [19, p. 283].

\section{B. Transmitter Model}

Typically, a transmitter includes some form of transmitter power control, which can be represented by the matrix equation [20]

$$
\tilde{y}=Q x
$$

which is equivalent to $\boldsymbol{y}=\boldsymbol{V} \boldsymbol{Q} \boldsymbol{x}$, where $\boldsymbol{Q}$ is the power control matrix, and $\boldsymbol{x}$ is a vector of complex source symbols (see Fig. 1). The linear precoder formed by $\boldsymbol{V} \boldsymbol{Q}$ [20], [21] introduces correlation between the symbols. We assume that $\boldsymbol{Q} \in \mathbb{R}^{n \times n}$ and $\boldsymbol{x} \in \mathbb{C}^{n}$ with its last $n-w$ entries equal to zero, where $w=\operatorname{rank}(\boldsymbol{Q}) \leq \operatorname{rank}(\boldsymbol{A})$ is the number of symbols actually transmitted within $n$ symbol intervals. Furthermore, we let the entries of $\boldsymbol{x}$ be independent and identically distributed (i.i.d.) random variables with unit variance. This does not restrict our transmitter model in any way because the required transmission energy is achieved by properly scaling the entries of $\boldsymbol{Q}$. Any channel coding, including space-time coding, is excluded from our system model.

In general, the power control matrix $\boldsymbol{Q}$ may be nondiagonal. If the entries of $\boldsymbol{x}$ are normal and our aim is to maximize channel capacity, the matrix $Q$ is diagonal [22]. In addition, in some simple suboptimal schemes for discrete signal constellations, the matrix $Q$ is diagonal [23]. Here, $Q$ is assumed to be a diagonal matrix whose entries are some function $f: \mathbb{R}^{n} \rightarrow \mathbb{R}$ of the energy gains $\lambda_{i}$ of the orthogonal subchannels, i.e., $q_{i i}=f\left(\lambda_{1}, \ldots, \lambda_{n}\right), 1 \leq i \leq n$.

A number of adaptive power control rules, together with corresponding mappings $f$, can be used in practical commu- nication systems. For example, with the water-filling power control rule, the transmitted signal is controlled according to [9, p. 2827], [24]

$$
q_{i i}=\sqrt{\left(\mu_{\mathrm{wf}}^{-1}-\lambda_{i}^{-1}\right)^{+}}, \quad 1 \leq i \leq n .
$$

On the other hand, with truncated power inversion, the transmitted signal is controlled according to [9, p. 2629]

$$
q_{i i}= \begin{cases}\sqrt{\beta / \lambda_{i}}, & \lambda_{i}>\mu_{\mathrm{tci}} \\ 0, & \lambda_{i} \leq \mu_{\mathrm{tci}} .\end{cases}
$$

Furthermore, with a simple power control method, sometimes referred to as downlink beamforming [25], only the $u$ th subchannel (that having the largest energy gain) is used for transmission, i.e.,

$$
q_{i i}= \begin{cases}\delta, & i=u \\ 0, & i \neq u\end{cases}
$$

The scalars $\beta$ and $\delta$ and the cutoff values or transmission thresholds $\mu_{\mathrm{wf}}$ and $\mu_{\mathrm{tci}}$ are chosen such that the long-term average energy or power constraint is fulfilled [20, p. 2279]. In general, the average power constraint takes a simpler form because the average transmitted power per symbol $P_{\mathrm{av}}$ is

$$
P_{\mathrm{av}}=\frac{\mathbb{E}\left[E_{\mathrm{tx}}\right]}{n T_{s}}=\frac{\mathbb{E}\left[\tilde{\boldsymbol{y}}^{*} \tilde{\boldsymbol{y}}\right]}{n T_{s}}=\frac{\mathbb{E}\left[\boldsymbol{x}^{*} \boldsymbol{Q}^{*} \boldsymbol{Q} \boldsymbol{x}\right]}{n T_{s}}
$$

where $T_{s}$ is the sampling interval. On the other hand, the average transmitted energy per symbol $E_{\text {av }}$ is

$$
E_{\mathrm{av}}=\mathbb{E}\left[\frac{E_{\mathrm{tx}}}{w}\right]=\mathbb{E}\left[\frac{\tilde{\boldsymbol{y}}^{*} \tilde{\boldsymbol{y}}}{w}\right]=\mathbb{E}\left[\frac{\boldsymbol{x}^{*} \boldsymbol{Q}^{*} \boldsymbol{Q} \boldsymbol{x}}{w}\right]
$$

because energy expenditure takes place only when it is used for transmission. The number $w$ of symbols actually transmitted can be a fixed number or a random variable. If the channel is time invariant or the power-control rule is such that the rank of $Q$ remains constant, then there is a simple relationship between the average transmitted power and energy. On the other hand, $w$ is likely to be a random variable when a power-control scheme with a transmission threshold $\mu$ is used. Hence, the exact relationship between average transmitted power and energy is difficult to establish. For those reasons, unless otherwise specified, in the remainder of this paper, we will consider only power-limited systems due to their analytical tractability. For a random $w$, the probability of outage or no transmission is

$$
P_{\text {out }}=\operatorname{Pr}(w=0) .
$$

\section{BASIC CONCEPTS}

\section{A. Energy Gain of the Channel}

The energy gain of the channel is the ratio of the signal component of the received energy $E_{\mathrm{rx}}$ to the transmitted energy $E_{\mathrm{tx}}$ and is given by

$$
G=\frac{E_{\mathrm{rx}}}{E_{\mathrm{tx}}}=\frac{\boldsymbol{z}^{*} \boldsymbol{z}}{\boldsymbol{y}^{*} \boldsymbol{y}}=\frac{\boldsymbol{y}^{*} \boldsymbol{H}^{*} \boldsymbol{H} \boldsymbol{y}}{\boldsymbol{y}^{*} \boldsymbol{y}}
$$


when $E_{\mathrm{tx}}>0$. On the other hand, when $E_{\mathrm{tx}}=0$, no transmission takes place, and the energy gain of the channel is defined to be zero.

The energy gain $G$ is in fact a Rayleigh quotient for an $n \times n$ Hermitian matrix $\boldsymbol{A}=\boldsymbol{H}^{*} \boldsymbol{H}$ [19, p. 282]. The form of the Rayleigh quotient is simplified if we use the virtual channel model [15]-[18] and take advantage of the invariance property of the Rayleigh quotient under unitary similarity transformations to obtain

$$
G=\frac{\boldsymbol{z}^{*} \boldsymbol{z}}{\boldsymbol{y}^{*} \boldsymbol{y}}=\frac{\tilde{\boldsymbol{z}}^{*} \tilde{\boldsymbol{z}}}{\tilde{\boldsymbol{y}}^{*} \tilde{\boldsymbol{y}}}=\frac{\tilde{\boldsymbol{y}}^{*} \boldsymbol{D}^{*} \boldsymbol{D} \tilde{\boldsymbol{y}}}{\tilde{\boldsymbol{y}}^{*} \tilde{\boldsymbol{y}}}=\frac{\sum_{i=1}^{n} \lambda_{i}\left|\tilde{y}_{i}\right|^{2}}{\sum_{i=1}^{n}\left|\tilde{y}_{i}\right|^{2}}
$$

where $\tilde{y}_{i}$ is the $i$ th element of vector $\tilde{\boldsymbol{y}}$. Let

$$
\varepsilon_{i}=\frac{\left|\tilde{y}_{i}\right|^{2}}{\sum_{i=1}^{n}\left|\tilde{y}_{i}\right|^{2}}, \quad i=1,2, \ldots, n
$$

denote the fraction of the total transmitted energy allocated to the $i$ th subchannel. Then, (13) can be rewritten as

$$
G=\sum_{i=1}^{n} \lambda_{i} \varepsilon_{i}
$$

which shows that the energy gain $G$ depends on the distribution of the available energy among subchannels rather than its total amount. Furthermore, by the properties of a Rayleigh quotient [19, p. 285], the energy gain of the channel $G$ is bounded as

$$
\lambda_{l} \leq G \leq \lambda_{u}
$$

where $\lambda_{l}$ and $\lambda_{u}$ are the minimal and the maximal eigenvalues of $\boldsymbol{A}$, respectively.

\section{B. Average Energy Gain of the Channel}

In general, the average energy gain of the channel is

$$
\bar{G}=\mathbb{E}[G]=\mathbb{E}\left[E_{\mathrm{rx}} / E_{\mathrm{tx}}\right]
$$

which is equal to $\mathbb{E}\left[E_{\mathrm{rx}}\right] / \mathbb{E}\left[E_{\mathrm{tx}}\right]$ if and only if $E_{\mathrm{tx}}$ and $G$ are uncorrelated, for example, if $E_{\mathrm{tx}}$ is fixed. By substituting (15) into (17), we obtain

$$
\bar{G}=\sum_{i=1}^{n} \mathbb{E}\left[\lambda_{i} \varepsilon_{i}\right]=\breve{G}+\theta .
$$

The parameter

$$
\breve{G}=\sum_{i=1}^{n} \mathbb{E}\left[\lambda_{i}\right] \mathbb{E}\left[\varepsilon_{i}\right]
$$

describes the average energy gain as if there was no correlation between the energy $\varepsilon_{i}$ allocated to the $i$ th subchannel and the subchannel energy gain $\lambda_{i}$. On the other hand, the term

$$
\theta=\sum_{i=1}^{n} \operatorname{Cov}\left[\lambda_{i}, \varepsilon_{i}\right]
$$

in (18) describes how $\bar{G}$ changes when there is correlation between $\varepsilon_{i}$ and $\lambda_{i}$.

\section{Representative Energy Gain of the Channel}

Our aim now is to find a representative energy gain of the channel that does not depend on the transmitted signal as do the energy gain in (13) and the average energy gain in (18). The average energy gain of (18) is independent of the transmitted signal if and only if the transmitted energy is uniformly distributed over all orthogonal subchannels associated with eigenvalues of the channel matrix, that is, $\varepsilon_{i}=1 / n$ for all $i$. Thus, the representative energy gain is uniquely defined to be

$$
G_{0}=\frac{1}{n} \mathbb{E}\left[\sum_{i=1}^{n} \lambda_{i}\right]=\frac{1}{n} \mathbb{E}[\operatorname{tr}(\boldsymbol{A})] .
$$

The representative energy gain describes the average attenuation of the channel for a special transmitted signal. The definition in (21) is consistent with and is in fact a generalization of the one used, for example, in [11] in a frequency-selective channel. In fact, the representative energy gain is the energy of the impulse response of the channel [11]. In frequency-selective time-invariant or frequency-selective block-fading channels, distinct frequencies create orthogonal subchannels. In multipleantenna channels, orthogonal subchannels are created by beamforming matrices. Thus, a uniform energy distribution over the orthogonal subchannels implies a uniform energy distribution across both the frequency and spatial dimensions.

\section{Average Transmitted and Received Energies}

The average transmitted energy is

$$
\mathbb{E}\left[E_{\mathrm{tx}}\right]=\mathbb{E}\left[\sum_{i=1}^{n}\left|\tilde{y}_{i}\right|^{2}\right]
$$

whereas the average received energy is

$$
\mathbb{E}\left[E_{\mathrm{rx}}\right]=\mathbb{E}\left[\sum_{i=1}^{n} \lambda_{i}\left|\tilde{y}_{i}\right|^{2}\right] .
$$

When water filling, truncated channel inversion, or downlink beamforming are used, the power control matrix $Q$ is diagonal. Then, the average transmitted and received energies, respectively, become

$$
\begin{aligned}
& \mathbb{E}\left[E_{\mathrm{tx}}\right]=\mathbb{E}\left[\sum_{i=1}^{u} q_{i i}^{2}\left|x_{i}\right|^{2}\right]=\mathbb{E}\left[\sum_{i=1}^{u} q_{i i}^{2}\right] \\
& \mathbb{E}\left[E_{\mathrm{rx}}\right]=\mathbb{E}\left[\sum_{i=1}^{u} \lambda_{i} q_{i i}^{2}\left|x_{i}\right|^{2}\right]=\mathbb{E}\left[\sum_{i=1}^{u} \lambda_{i} q_{i i}^{2}\right] .
\end{aligned}
$$

To obtain the simplified forms of (24) and (25), we take advantage of the assumption that the entries of $\boldsymbol{x}$ are i.i.d. random variables with unit variance.

In the most general case, $q_{i i}^{2}$ follows a mapping $g: \mathbb{R}^{n} \rightarrow$ $\mathbb{R}$, and the evaluation of (24) and (25) requires averaging over the joint probability density function (pdf) $p\left(\lambda_{1}, \ldots, \lambda_{n}\right)$ of the eigenvalues of $\boldsymbol{A}$. However, in the special case where the $i$ th 
diagonal entry $q_{i i}$ is a function only of $\lambda_{i}$, i.e., $g:[0, \infty) \rightarrow \mathbb{R}$, we have

$$
\begin{aligned}
& \mathbb{E}\left[E_{\mathrm{tx}}\right]=\mathbb{E}\left[\sum_{i=1}^{u} g\left(\lambda_{i}\right)\right]=u \int_{0}^{\infty} g(\lambda) h(\lambda) d \lambda \\
& \mathbb{E}\left[E_{\mathrm{rx}}\right]=\mathbb{E}\left[\sum_{i=1}^{u} \lambda_{i} g\left(\lambda_{i}\right)\right]=u \int_{0}^{\infty} \lambda g(\lambda) h(\lambda) d \lambda
\end{aligned}
$$

where $h(\lambda)$ is the pdf of a single eigenvalue [26]. It can easily be verified that, under the long-term average energy or power constraint $\left[20\right.$, p. 2279], the scalars $\beta, \delta, \mu_{\mathrm{wf}}$, and $\mu_{\mathrm{tci}}$ depend on the specific joint distribution of eigenvalues $\left(\lambda_{1}, \ldots, \lambda_{n}\right)$ rather than their instantaneous values. Consequently, water filling and the truncated channel inversion and downlink beamforming power-control schemes fall under this special case because, in all of them, $q_{i i}$, as (6)-(8) suggest, depends on only a single eigenvalue $\lambda_{i}$.

\section{E. Average Transmitted and Received SNRS}

The average transmitted and received energies are usually normalized by the receiver noise spectral density $N_{0}$, leading to the average transmitted SNR per symbol

$$
\bar{\gamma}_{\mathrm{tx}}=\frac{1}{n} \frac{\mathbb{E}\left[E_{\mathrm{tx}}\right]}{N_{0}}=\frac{1}{n} \frac{\mathbb{E}\left[\tilde{\boldsymbol{y}}^{*} \tilde{\boldsymbol{y}}\right]}{N_{0}}
$$

and the average received SNR per symbol

$$
\bar{\gamma}_{\mathrm{rx}}=\frac{1}{n} \frac{\mathbb{E}\left[E_{\mathrm{rx}}\right]}{N_{0}}=\frac{1}{n} \frac{\mathbb{E}\left[E_{\mathrm{tx}} \cdot G\right]}{N_{0}}=\frac{1}{n} \frac{\mathbb{E}\left[\tilde{\boldsymbol{z}}^{*} \tilde{z}\right]}{N_{0}} .
$$

The average transmitted SNR per symbol referred to the receiver is defined as

$$
\breve{\gamma}_{\mathrm{tx}}=\frac{1}{n} \frac{\mathbb{E}\left[E_{\mathrm{tx}}\right] \cdot G_{0}}{N_{0}}=\bar{\gamma}_{\mathrm{tx}} G_{0}
$$

where $G_{0}$ is given by (21). The averages in (28)-(30) include outages. In [6, eq. (13)], the SNR corresponding to (30) was called the average transmitted energy-to-noise ratio. To avoid confusion, we have reserved the term average transmitted SNR per symbol for (28) because of the scaling by $G_{0}$ in (30). The scaling is used for convenience to take into account the average attenuation of the channel for a signal having a uniform distribution in frequency and spatial dimensions.

The relationship between the average transmitted SNR per symbol $\bar{\gamma}_{\text {tx }}$, the average transmitted SNR per symbol referred to the receiver $\breve{\gamma}_{t x}$, and the average received SNR per symbol $\bar{\gamma}_{\mathrm{rx}}$ can be established through the covariance of the transmitted energy $E_{\mathrm{tx}}$ and channel energy gain $G$. In particular

$$
\bar{\gamma}_{\mathrm{rx}}=\bar{\gamma}_{\mathrm{tx}} \mathbb{E}[G]+\frac{\vartheta}{n N_{0}}=\frac{\breve{\gamma}_{\mathrm{tx}}}{G_{0}} \mathbb{E}[G]+\frac{\vartheta}{n N_{0}}
$$

where

$$
\vartheta=\operatorname{Cov}\left[E_{\mathrm{tx}}, G\right]=\mathbb{E}\left[E_{\mathrm{tx}} \cdot G\right]-\mathbb{E}\left[E_{\mathrm{tx}}\right] \cdot \mathbb{E}[G] .
$$

Mathematically, the covariance $\vartheta$ can be bounded as

$$
-\sqrt{\operatorname{Var}\left[E_{\mathrm{tx}}\right] \operatorname{Var}[G]} \leq \vartheta \leq \sqrt{\operatorname{Var}\left[E_{\mathrm{tx}}\right] \operatorname{Var}[G]} .
$$

However, in the present system model, there is an alternative lower bound on the covariance. It is obtained by imposing the physical constraint on the received energy that it cannot be negative so that $\mathbb{E}\left[E_{\mathrm{tx}} \cdot G\right] \geq 0$. After some algebra in (32), we obtain $\vartheta \geq-\mathbb{E}\left[E_{\mathrm{tx}}\right] \cdot \mathbb{E}[G]$. Finally, the lower bound for the covariance is given by

$$
\vartheta \geq-\min \left\{\mathbb{E}\left[E_{\mathrm{tx}}\right] \mathbb{E}[G], \sqrt{\operatorname{Var}\left[E_{\mathrm{tx}}\right] \operatorname{Var}[G]}\right\} .
$$

By substituting (18) into (31), we obtain

$$
\bar{\gamma}_{\mathrm{rx}}=\bar{\gamma}_{\mathrm{tx}}(\breve{G}+\theta)+\frac{\vartheta}{n N_{0}}=\frac{\breve{\gamma}_{\mathrm{tx}}}{G_{0}}(\breve{G}+\theta)+\frac{\vartheta}{n N_{0}} .
$$

To summarize, we have defined three different averages of the energy gain $G$. The statistical average (17) is denoted by $\bar{G}$. If $\theta=0$ in (18), we obtain $\bar{G}=\breve{G}$. If, in addition, $\vartheta=0$ in (35), we obtain $\bar{G}=G_{0}$. The covariance $\vartheta$ specifies how well the transmitted energy is statistically matched to the energy gain of the channel. The covariance $\theta$ specifies how well the energy allocations $\varepsilon_{i}$ are statistically matched to the energy gains $\lambda_{i}$ of the orthogonal subchannels. The covariances $\vartheta$ and $\theta$ describe the change of $\bar{\gamma}_{\mathrm{rx}}$ due to power control in the transmitter. Since they can take negative and positive values, $\bar{\gamma}_{\mathrm{rx}}$ could be smaller or greater than $\breve{G} \bar{\gamma}_{\text {tx }}$. The upper and lower bounds on covariance $\vartheta$, which lead to the respective bounds on $\bar{\gamma}_{\mathrm{rx}}$ with respect to $\bar{\gamma}_{\mathrm{tx}}(\breve{G}+\theta)$, can be used in link budget calculations for adaptive links.

We want to emphasize that it would be misleading to refer to (30) as the average received SNR per symbol because the covariances $\theta$ and $\vartheta$ in (35) are, in general, nonzero and depend on the transmitted signal. Furthermore, if we want to know how efficiently the basic resource, i.e., transmitted energy, is used, we should not use (29) instead of (30) in performance comparisons.

So far, all the SNRs are presented as if the system were power limited. In energy-limited systems, we use the expurgated SNRs

$$
\begin{aligned}
& \breve{\gamma}_{\mathrm{tx}}^{(e)}=\frac{1}{n} \frac{\mathbb{E}\left[E_{\mathrm{tx}} \mid E_{\mathrm{tx}}>0\right] \cdot G_{0}}{N_{0}}=\frac{\breve{\gamma}_{\mathrm{tx}}}{1-P_{\mathrm{out}}} \\
& \bar{\gamma}_{\mathrm{rx}}^{(e)}=\frac{1}{n} \frac{\mathbb{E}\left[E_{\mathrm{tx}} \cdot G \mid E_{\mathrm{tx}} \cdot G>0\right]}{N_{0}}=\frac{\bar{\gamma}_{\mathrm{rx}}}{1-P_{\mathrm{out}}}
\end{aligned}
$$

where the probability of outage $P_{\text {out }}$ is defined in (11). In a similar way, the transmitted SNR in (28) can be expurgated. A summary of the various SNRs is given in Table I. We have borrowed the term "expurgation" from the channel-coding literature [27]. The purpose of this expurgation process is to remove the effect of outage from the calculation of the average SNR values of (36) and (37). That is, we remove the effect of not transmitting during outage from the average SNR calculation. 
TABLE I

Summary of the Different SNR Concepts USEd IN THIS PAPER

\begin{tabular}{ccc}
\hline Name & Nonexpurgated & Expurgated \\
\hline Average transmitted SNR & $\bar{\gamma}_{\mathrm{tx}}=\frac{1}{n} \frac{\mathbb{E}\left[E_{\mathrm{tx}}\right]}{N_{0}}$ & $\bar{\gamma}_{\mathrm{tx}}^{(e)}=\frac{1}{n} \frac{\mathbb{E}\left[E_{\mathrm{tx}} \mid E_{\mathrm{tx}}>0\right]}{N_{0}}$ \\
Average received SNR & $\bar{\gamma}_{\mathrm{rx}}=\frac{1}{n} \frac{\mathbb{E}\left[E_{\mathrm{tx}} \cdot G\right]}{N_{0}}$ & $\bar{\gamma}_{\mathrm{rx}}^{(e)}=\frac{1}{n} \frac{\mathbb{E}\left[E_{\mathrm{tx}} \cdot G \mid E_{\mathrm{tx}} \cdot G>0\right]}{N_{0}}$ \\
Average transmitted SNR referred to the receiver & $\breve{\gamma}_{\mathrm{tx}}=\frac{1}{n} \frac{\mathbb{E}\left[E_{\mathrm{tx}}\right] \cdot G_{0}}{N_{0}}$ & $\breve{\gamma}_{\mathrm{tx}}^{(e)}=\frac{1}{n} \frac{\mathbb{E}\left[E_{\mathrm{tx}} \mid E_{\mathrm{tx}}>0\right] \cdot G_{0}}{N_{0}}$ \\
\hline
\end{tabular}

\section{EXAMPLES}

\section{A. Frequency-Nonselective Fading Channel}

We consider a frequency-nonselective fading channel with $t$ transmitter and $r$ receiver antennas. The number of channel inputs $n$ is equal to $t$, and the number of channel outputs $m$ is equal to $r$. Furthermore, we assume that the entries of the channel matrix $\boldsymbol{H}$ are i.i.d. circularly symmetric complex Gaussian random variables with zero mean and variance $\sigma^{2}$. Consequently, the $n \times n$ matrix $\boldsymbol{A}=\boldsymbol{H}^{*} \boldsymbol{H}$ is a complex Wishart matrix, and the pdf of a single unordered eigenvalue of $\boldsymbol{A}$ is [28, eq. (15)]

$$
h(\lambda)=\frac{e^{-\lambda / \sigma^{2}}}{u \sigma^{2}} \sum_{k=0}^{u-1} \frac{k !}{(k+\alpha) !}\left(\frac{\lambda}{\sigma^{2}}\right)^{\alpha}\left[L_{k}^{(\alpha)}\left(\frac{\lambda}{\sigma^{2}}\right)\right]^{2}
$$

where $u=\min (m, n), \quad v=\max (m, n), \quad \alpha=v-u$, and $L_{k}^{(\alpha)}(x)$ is the associated Laguerre polynomial of order $\alpha$. The cumulative distribution function (cdf) of the largest eigenvalue $F_{\lambda}(x)$ is [29, p. 421]

$$
F_{\lambda}(x)=\frac{\Gamma_{u}(u)}{\Gamma_{u}(u+v)}\left(\frac{x}{\sigma^{2}}\right)^{u v}{ }_{1} \mathrm{~F}_{1}\left(v ; u+v ;-\frac{x}{\sigma^{2}} \boldsymbol{I}_{u}\right)
$$

where $\Gamma_{u}(\cdot)$ is the complex multivariate Gamma function [30, eq. (83)], and ${ }_{p} \mathrm{~F}_{q}(\cdot ; \cdot ; \cdot)$ is the complex hypergeometric function of a matrix argument [30, eq. (87)]. These functions can efficiently be evaluated using algorithms developed in [31]. The representative energy gain of the channel is given by

$$
G_{0}=\frac{1}{n} \mathbb{E}[\operatorname{tr}(\boldsymbol{A})]=\frac{1}{n} m n \sigma^{2}=m \sigma^{2} .
$$

A frequency-nonselective fading channel with a single transmitter and single receiver antennas is a special case of a frequency-nonselective fading channel where the channel matrix $\boldsymbol{H}$ is a random scalar. The entry $h_{11}$ simply describes the channel response at a given time instant, and $\lambda_{1}=a_{11}=\left|h_{11}\right|^{2}$.

In our simulations, we use a channel whose fading gain is represented by the sum [32]-[34]

$$
h_{11}=\frac{1}{N} \sum_{n=0}^{N-1} \exp \left(\sqrt{-1} \psi_{n}\right)
$$

where $N$ is the number of complex equal-amplitude subpaths, and $\psi_{n}$ is the phase of the $n$th subpath. If the phases are all equal, the sum (41) is a coherent sum whose magnitude is equal to unity. Thus, peak normalization is used in (41). The amplitudes of the subpaths in (41) are identical, which is only a convenient selection for our numerical results [32].

If the phases $\psi_{n}$ in (41) are random, independent, and uniformly distributed, (41) corresponds to a noncoherent sum. The pdf of the magnitude of (41) can be derived from the results presented in [34] for the values $N=2$ and 3. For large $N$, it can be approximated with a truncated Rayleigh distribution. The pdf of the squared magnitude of (41) can be derived from the work of [32]. For large $N$, it can be approximated with a truncated exponential distribution. Its peak value is unity, and the average value is $1 / N$. We can alternatively use average normalization. In that case, we replace $1 / N$ in (41) by $1 / \sqrt{N}$.

\section{B. Frequency-Selective Block-Fading Channel}

We next consider a frequency-selective fading channel with $t$ transmitter and $r$ receiver antennas. We assume that the matrix-valued channel impulse response is finite and spans $L+1<\infty$ symbol intervals. Furthermore, we assume that the channel is quasi-static ${ }^{1}$ for $K \gg L+1$ symbol intervals. Finally, we assume that symbols are transmitted in blocks of $K$ symbols and that there is no interblock interference.

Let $\boldsymbol{y}_{k} \in \mathbb{C}^{t}$ and $\boldsymbol{z}_{k} \in \mathbb{C}^{r}$ denote the transmitted and received vectors, respectively, at time instant $k$. The vector of received symbols at the $k$ th time instant is then given as

$$
\boldsymbol{z}_{k}=\sum_{l=0}^{L} \boldsymbol{H}_{l} \boldsymbol{y}_{k-l}
$$

where $\boldsymbol{H}_{l}$ is a matrix-valued channel model corresponding to the $l$ th tap. We assume that the matrices $\boldsymbol{H}_{l}$ are independently distributed complex Gaussian matrices with variance $\sigma_{l}^{2}$. By stacking the vectors $\boldsymbol{y}_{k}$ and $\boldsymbol{z}_{k}$, we obtain (43), shown at the bottom of the next page, where $\boldsymbol{H} \in \mathbb{C}^{K r \times K t}$ is a blockToeplitz convolution matrix.

Block-Toeplitz matrices are asymptotically equivalent to block-circulant matrices, which implies that the eigenvalues of a block-Toeplitz matrix and a properly constructed blockcirculant matrix asymptotically converge [35]. Since a blockcirculant matrix can be block diagonalized by a block-Fourier matrix, the eigenvalues of the block-Toeplitz matrix can easily be approximated [35]. More precisely, for a sufficiently large ratio $K / L \gg 1$, we obtain

$$
\boldsymbol{H} \approx\left(\boldsymbol{F}_{K} \otimes \boldsymbol{I}_{r}\right)^{*} \boldsymbol{D}\left(\boldsymbol{F}_{K} \otimes \boldsymbol{I}_{t}\right)
$$

\footnotetext{
${ }^{1}$ That is, it remains constant for $K$ symbol intervals and randomly changes from block to block.
} 
where $\otimes$ denotes the Kronecker product, $\boldsymbol{F}_{K}$ is the $K \times K$ Fourier matrix

$$
\boldsymbol{F}_{K}=\frac{1}{\sqrt{K}}\left[\omega^{(i-1)(j-1)}\right]_{i, j=1}^{K, K}
$$

with $\omega=e^{-2 \pi \sqrt{-1} / K}$, and $\boldsymbol{D} \in \mathbb{C}^{K r \times K t}$ is a block-diagonal matrix $\quad \boldsymbol{D}=\operatorname{diag}\left(\boldsymbol{D}_{0}, \boldsymbol{D}_{1}, \ldots, \boldsymbol{D}_{K-1}\right) \quad$ with $\quad \boldsymbol{D}_{k} \in \mathbb{C}^{r \times t}$ given as

$$
\boldsymbol{D}_{k}=\sum_{l=0}^{L} \omega^{k l} \boldsymbol{H}_{l}
$$

The entries of $\boldsymbol{D}_{k}$ are zero-mean complex Gaussian random variables as are the entries of $\boldsymbol{H}_{l}$. The variance of the entries of $\boldsymbol{D}_{k}$ is the sum of variances of respective entries of $\boldsymbol{H}_{l}$, i.e., $\sigma^{2}=\sum_{l=0}^{L} \sigma_{l}^{2}$.

The joint pdf of the unordered eigenvalues of $\boldsymbol{A}=$ $\operatorname{diag}\left(\boldsymbol{A}_{0}, \ldots, \boldsymbol{A}_{K-1}\right)$, where $\boldsymbol{A}_{k}=\boldsymbol{D}_{k}^{*} \boldsymbol{D}_{k}$ is a complex Wishart matrix, is unknown [36]. This precludes the derivation of the cdf of the largest eigenvalue. However, the average received energy can still be found approximately as

$$
\begin{aligned}
\mathbb{E}\left[E_{\mathrm{rx}}\right] & \approx \sum_{i=1}^{K u} \int_{0}^{\infty} \ldots \int_{0}^{\infty} \lambda_{i} g\left(\lambda_{i}\right) p\left(\lambda_{1}, \ldots, \lambda_{K u}\right) d \lambda_{1} \cdots d \lambda_{K u} \\
& =K \sum_{i=1}^{u} \int_{0}^{\infty} \cdots \int_{0}^{\infty} \lambda_{i} g\left(\lambda_{i}\right) p\left(\lambda_{1}, \ldots, \lambda_{u}\right) d \lambda_{1} \cdots d \lambda_{u} \\
& =K u \int_{0}^{\infty} \lambda g(\lambda) h(\lambda) d \lambda
\end{aligned}
$$

where $u=\min (r, t), v=\max (r, t)$, and $h(\lambda)$ is given by (38). The average transmitted energy can be obtained in a similar way. The representative energy gain of the channel is then

$$
G_{0} \approx \frac{\mathbb{E}[\operatorname{tr}(\boldsymbol{A})]}{K t}=\frac{1}{K t} \mathbb{E}\left[\sum_{k=0}^{K-1} \operatorname{tr}\left(\boldsymbol{A}_{k}\right)\right]=r \sum_{l=0}^{L} \sigma_{l}^{2} .
$$

A frequency-selective block-fading channel with a single transmitter and single receiver antennas is a special case of a frequency-selective fading channel where $t=r=1$. Consequently, $\boldsymbol{H} \in \mathbb{C}^{K \times K}$ is a Toeplitz matrix with the entry $h_{i j}$ being the $j$ th sample of the channel impulse response at the $i$ th time instant.

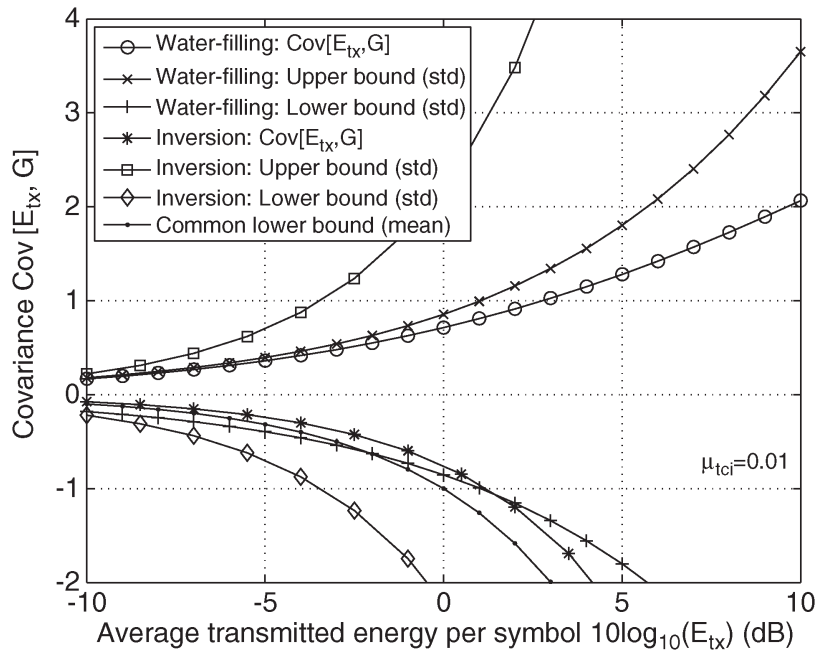

Fig. 2. Upper and lower bounds on the covariance value in a single-antenna channel with Rayleigh fading and $\sigma^{2}=1$.

\section{Numerical Results}

\section{A. Covariance}

The covariances in (35) are a measure of how well the transmitted signal is matched in a statistical sense to the channel: The larger the covariances $\theta$ and $\vartheta$, the larger the ratio $\bar{\gamma}_{\mathrm{rx}} / \bar{\gamma}_{\mathrm{tx}}$. In Fig. 2, we show a comparison of water filling and truncated channel inversion in a single-antenna channel with Rayleigh fading and $\sigma^{2}=1$. Since, in any single-antenna channel $\theta=$ 0 , the power control methods are compared according to the achievable covariance value $\vartheta$. The upper bound (33) and the lower bound (34) of the covariance are plotted for comparison.

The numerical results suggest that, in a single-antenna Rayleigh fading channel with low-power transmission, one approaches the covariance upper limit with water filling. On the other hand, with truncated channel inversion, one operates close to the covariance lower limit. In other words, water filling gives almost the highest possible average received SNR $\bar{\gamma}_{\mathrm{rx}}$, whereas truncated channel inversion gives almost the lowest possible average received SNR $\bar{\gamma}_{\mathrm{rx}}$, provided that $\bar{\gamma}_{\mathrm{tx}}$ and $G_{0}$ are kept constant in comparisons. Truncated channel inversion remains useful because performance is improved by keeping the received SNR constant during transmission.

We plot the ratio $\bar{\gamma}_{\text {rx }} / \breve{\gamma}_{\text {tx }}$ that can be achieved in a frequencynonselective or a frequency-selective MIMO channel with water filling and truncated channel inversion in Figs. 3 and 4, respectively. The numerical results show that $\bar{\gamma}_{\mathrm{rx}}$ is larger than $\breve{\gamma}_{\text {tx }}$ when water filling is used. On the other hand, if truncated

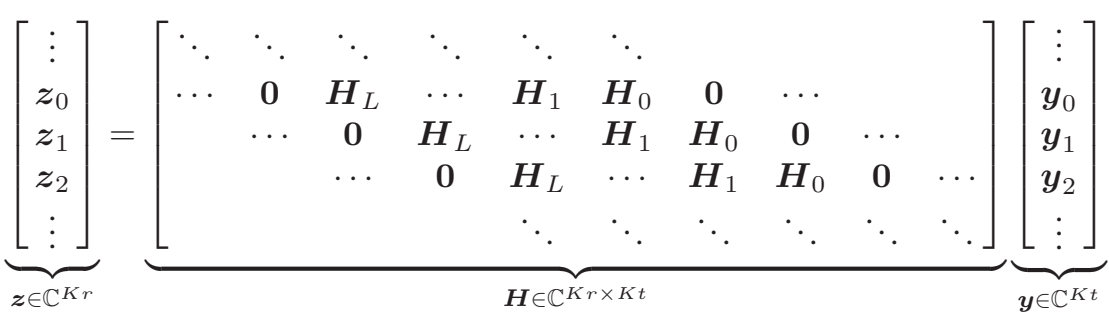




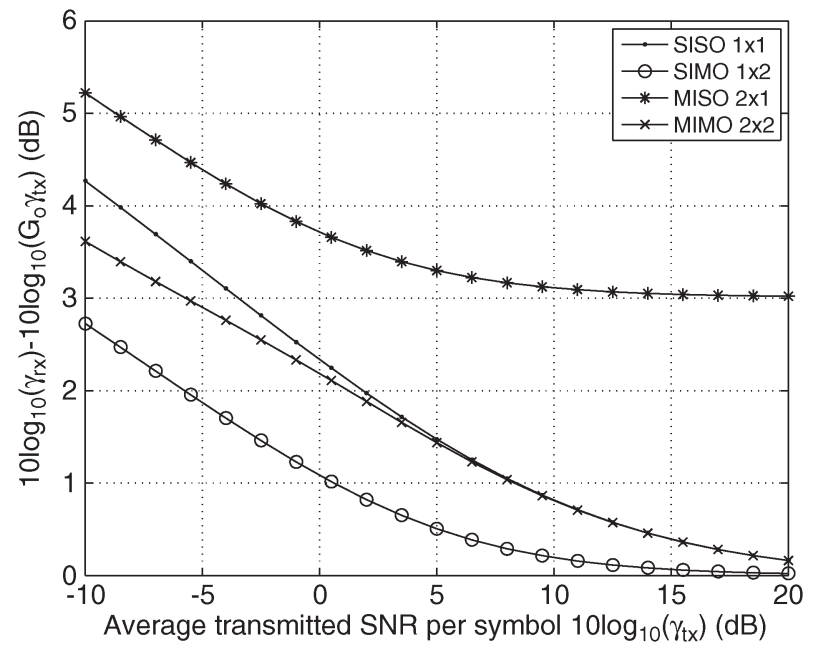

Fig. 3. Relationship between the average received SNR $\bar{\gamma}_{\mathrm{rx}}$ and the average transmitted SNR referred to the receiver $G_{0} \bar{\gamma}_{\text {tx }}$ in a multiantenna channel with water filling and $\sigma^{2}=1$.

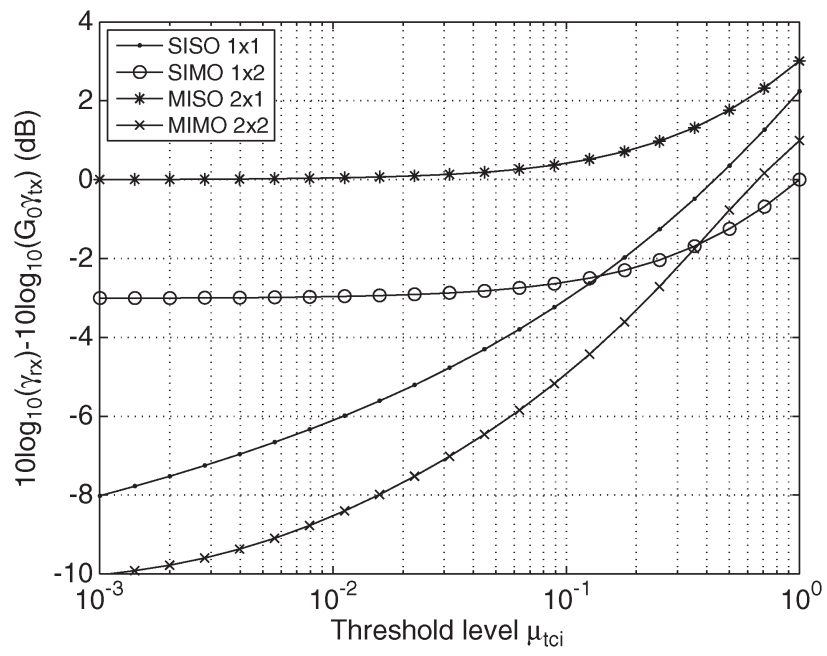

Fig. 4. Relationship between the average received SNR $\bar{\gamma}_{\mathrm{rx}}$ and the average transmitted SNR referred to the receiver $G_{0} \bar{\gamma}_{\text {tx }}$ in a multiantenna channel with truncated channel inversion and $\sigma^{2}=1$.

channel inversion is used, $\bar{\gamma}_{\mathrm{rx}}$ could be larger or smaller than $\breve{\gamma}_{\text {tx }}$, depending on the threshold $\mu_{\text {tci }}$. The relationship between $\bar{\gamma}_{\text {rx }}$ and $\breve{\gamma}_{\text {tx }}$ does not depend on the average transmitted SNR per symbol $\bar{\gamma}_{t x}$ when truncated channel inversion is used because $\beta$ cancels when dividing (27) by (26).

In a multiple-input-single-output (MISO) system with a two-transmitter/one-receiver antenna $(2 \times 1)$ and a singleinput-multiple-output (SIMO) system with one-transmitter/ two-receiver antennas $(1 \times 2)$, the average received SNR $\bar{\gamma}_{\mathrm{rx}}$ is actually the same. It is because there is only one orthogonal subchannel, which implies that $\theta=0$, and the distribution of the corresponding positive eigenvalue is the same in both cases. The difference in $\bar{\gamma}_{\mathrm{rx}} / \breve{\gamma}_{\mathrm{tx}}$ shown in Figs. 3 and 4 comes from the fact that the representative energy gain differs. Specifically, in a MISO $2 \times 1$ system, $G_{0}=1$, whereas in a SIMO $1 \times 2$ system, $G_{0}=2$. Since $\bar{\gamma}_{\mathrm{rx}}$ is equal to $\breve{\gamma}_{\mathrm{tx}}$ if $\theta=0$ and $\vartheta=0$, i.e., when there is no power control at the transmitter, the results in Figs. 3 and 4 demonstrate how beneficial power control is at the transmitter in a MISO system.

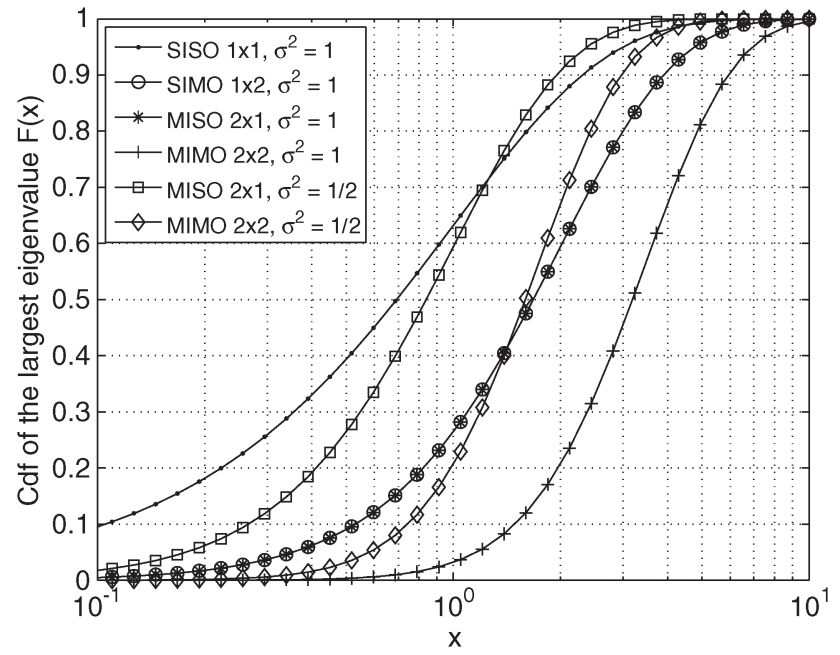

Fig. 5. Cdf of the largest eigenvalue $\lambda_{u}$ in a frequency-nonselective multiantenna channel.

The peak energy gain of the channel is bounded by the largest eigenvalue $\lambda_{u}$ of the matrix $\boldsymbol{A}$. The cdf of $\lambda_{u}$ in a number of frequency-nonselective multiantenna channels is shown in Fig. 5. For MISO and MIMO channels, we also plot the results in the case when average normalization is used, i.e., when $G_{0}=1$.

In general, the peak energy gain should be less than or equal to 1 to satisfy the energy-conservation law. The problem of the proper normalization of the peak energy gain does not normally arise if one includes path loss in the model, which scales down the maximum eigenvalue, and uses the average transmitted SNR $\bar{\gamma}_{\text {tx }}$ to compare different systems. However, a common practice is to use the average transmitted SNR referred to the receiver $\breve{\gamma}_{t x}$ or the average received SNR $\bar{\gamma}_{\mathrm{rx}}$ and compare different systems against each other or against a unit-gain additive white Gaussian noise (AWGN) channel.

The results in Fig. 5 suggest that, in all the considered channel models, there is a nonnegligible probability, even if the channels are normalized according to the representative energy gain, the peak energy gain exceeds 1 . Consequently, a comparison of the performance attained by an adaptive system in the presented channel models and in a unit-gain AWGN can lead to erroneous conclusions. This effect could be particularly visible in adaptive systems that are able to take advantage of $\lambda_{u}>1$.

A solution to the problem is to normalize the channel with respect to the peak energy gain. Unfortunately, this is not always possible because the peak energy gain could be unbounded as in the Rayleigh fading model. In this case, we propose to normalize the channel in a statistical sense, i.e., to normalize it such that the peak energy gain exceeds 1 only with some small probability $\zeta$. For instance, the actual value of $\zeta$ can be adopted from a "six sigma" rule in production quality assessment, where $\zeta=3.4 \cdot 10^{-6}[37]$.

\section{B. SNR Distributions}

We assume that both the receiver and the transmitter know the channel. The modulation method in the examples is binary antipodal. One sample is taken per symbol. We present the 


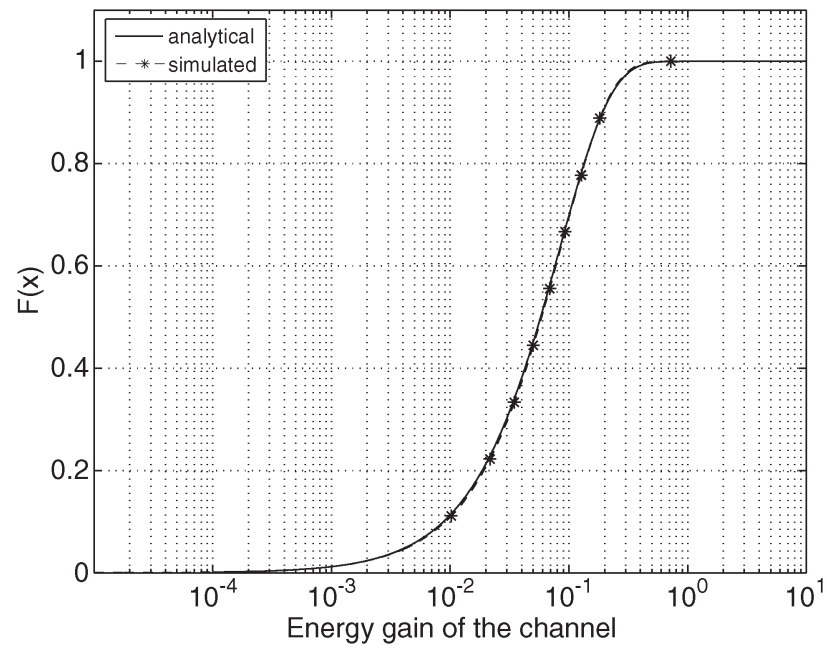

Fig. 6. Cdf of the energy gain of the channel.

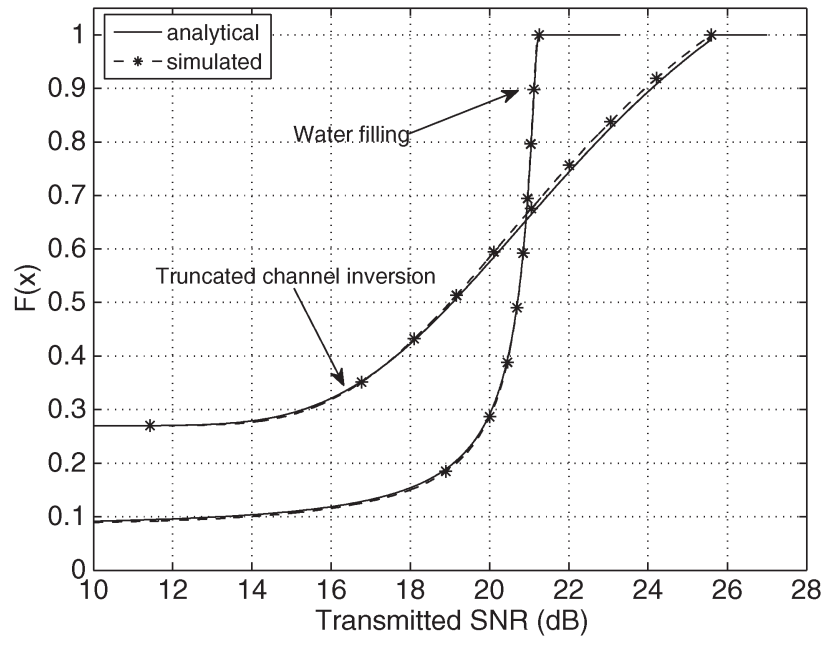

Fig. 7. Cdf of the transmitted SNR in water filling and truncated channel inversion.

analytical pdf's by assuming that the energy gain is exponentially distributed. We also present the simulated histograms by using the noncoherent sum of complex exponentials in (41) to represent a frequency-nonselective channel model. In the pdf's of the SNRs, there is an impulse at the origin corresponding to an outage with no transmission. When we include the impulse, the area under the pdf's is unity. We use the cdf's to illustrate the distributions.

In Fig. 6, we show the cdf of the energy gain of the channel when $E_{\mathrm{tx}}$ is always positive. The cdf of the exponential distribution has the form

$$
F(\lambda)=1-\exp \left(-\lambda / \sigma^{2}\right)
$$

for $\lambda \geq 0$, where $\lambda=\left|h_{11}\right|^{2}$. We can compare the cdf of (41) with peak normalization and (49) by setting $\sigma^{2}=1 / N$. If $N \gg 1$, the cdf's are almost identical, except for $\lambda>1$ [32]. If the transmission threshold is $\mu$, the probability of outage (11) for the exponential distribution is

$$
P_{\text {out }}=\operatorname{Pr}(\lambda<\mu)=1-\exp \left(-\mu / \sigma^{2}\right) .
$$

In Figs. 7 and 8, we present the cdf's for the transmitted and received SNRs for water filling and truncated channel

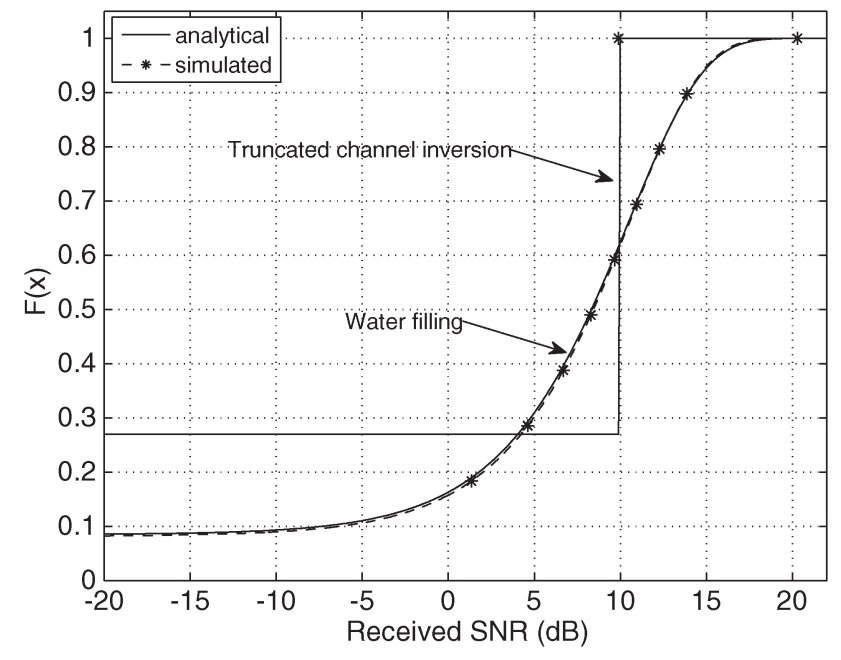

Fig. 8. Cdf of the received SNR in water filling and truncated channel inversion.

inversion when the average transmitted SNR is $20 \mathrm{~dB}$, that is, $10 \log _{10} \bar{\gamma}_{\mathrm{tx}}=20 \mathrm{~dB}$, and we assume that the noise power spectral density is unity. The transmission thresholds are selected for water filling and truncated channel inversion by using [38, eqs. (8) and (47)], respectively. When the average transmitted SNR is $20 \mathrm{~dB}$, the parameters are $\mu_{\mathrm{wf}}=0.0074$, $\mu_{\mathrm{tci}}=0.0271$, and $\beta=9.871$. The theoretical probability of outage for the exponential distribution is with these parameters $P_{\text {out }}=0.0853$ for water filling and $P_{\text {out }}=0.2780$ for truncated channel inversion. Our aim is not to minimize the bit error probability but to demonstrate how the different power control rules behave. Corresponding analytical results for the SNR distributions are obtained from the results presented in [39, pp. 90-104] by using (6), (7), and (49). These results are summarized in the following sections.

1) Transmitted SNR: The cdf of the transmitted SNR is shown in Fig. 7. In water filling, the cdf has the form

$$
F_{\mathrm{wf}}\left(\gamma_{\mathrm{tx}}\right)= \begin{cases}P_{\mathrm{out}}, & \text { for } \gamma_{\mathrm{tx}}=0 \\ F\left(\frac{\mu_{\mathrm{wf}}}{1-\gamma_{\mathrm{tx}} \mu_{\mathrm{wf}}}\right), & \text { for } 0<\gamma_{\mathrm{tx}} \leq \mu_{\mathrm{wf}}^{-1} \\ 1, & \text { for } \gamma_{\mathrm{tx}}>\mu_{\mathrm{wf}}^{-1}\end{cases}
$$

where $F(\cdot)$ is the cdf of the channel energy gain. In truncated channel inversion, the cdf is

$$
F_{\mathrm{tci}}\left(\gamma_{\mathrm{tx}}\right)= \begin{cases}P_{\mathrm{out}}, & \text { for } \gamma_{\mathrm{tx}}=0 \\ P_{\mathrm{out}}+1-F\left(\beta / \gamma_{\mathrm{tx}}\right), & \text { for } 0<\gamma_{\mathrm{tx}} \leq \beta / \mu_{\mathrm{tci}} \\ 1, & \text { for } \gamma_{\mathrm{tx}}>\beta / \mu_{\mathrm{tci}} .\end{cases}
$$

2) Received SNR: In Fig. 8, we show the cdf of the received SNR for water filling. The analytical cdf is

$$
F_{\mathrm{wf}}\left(\gamma_{\mathrm{rx}}\right)=F\left[\mu_{\mathrm{wf}}\left(\gamma_{\mathrm{rx}}+1\right) / \bar{\gamma}_{\mathrm{rx}}\right]
$$

where $\gamma_{\mathrm{rx}} \geq 0$. For truncated channel inversion, the cdf of the received SNR is

$$
F_{\mathrm{tci}}\left(\gamma_{\mathrm{rx}}\right)= \begin{cases}P_{\mathrm{out}}, & \text { for } \gamma_{\mathrm{rx}} \leq \beta \\ 1, & \text { for } \gamma_{\mathrm{rx}}>\beta\end{cases}
$$




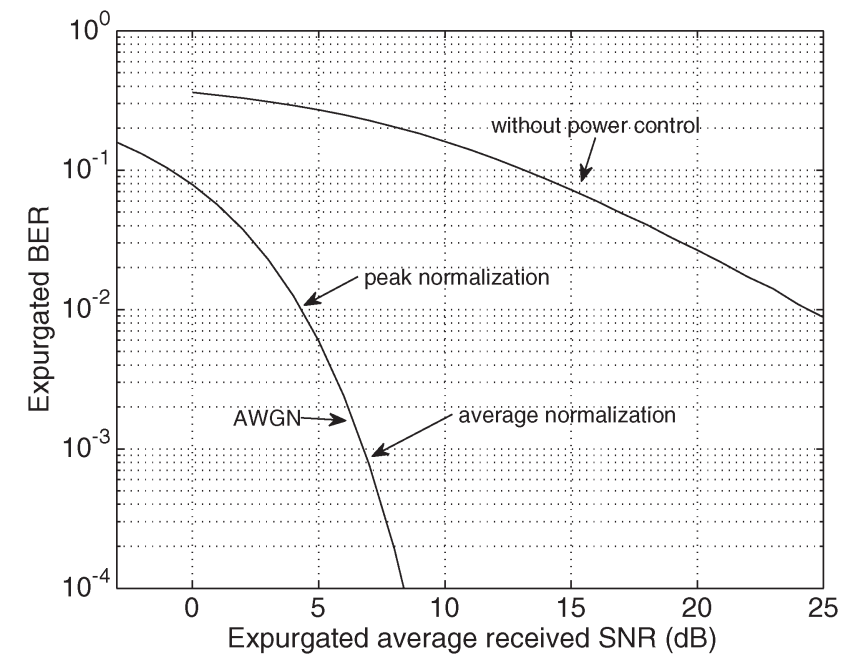

Fig. 9. Expurgated BER as a function of expurgated average received SNR per bit (truncated channel inversion).

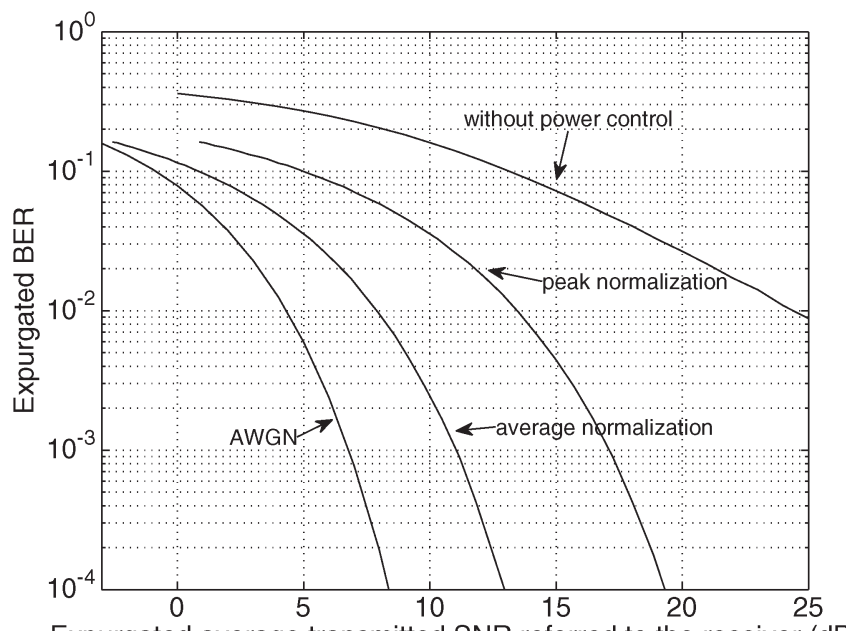

Expurgated average transmitted SNR referred to the receiver (dB)

Fig. 10. Expurgated BER as a function of expurgated average transmitted SNR per bit referred to the receiver (truncated channel inversion).

\section{BER With Power Control}

In our simulated system, the bit rate is constant above the threshold $\mu_{\text {tci }}$ in (7). We want to demonstrate how the biterror-rate (BER) performance should be presented in an energylimited system after we have analyzed the system as if it were power limited. The power-limited model has been used for mathematical tractability. We measure the BER only when we actually transmit energy in (7). Consequently, the average of the different SNRs is also measured under the condition that there is transmission. This implies that we use the expurgated SNRs defined in (36) and (37). Now, we are using the average energy actually transmitted per bit, but due to the outages, the average bit rates of different systems may be different, even in the same channel.

The BER performance for truncated channel inversion is presented in Figs. 9 and 10 by using either the expurgated average received SNR or the transmitted SNR referred to the receiver, respectively. We have used both peak and average normalization. In Fig. 9, the normalization method does not have any effect on the performance. One could conclude that no performance gain can be obtained, but Fig. 10 shows that significant gain is possible, for example, by using diversity. Since the channel is assumed to be known, there is no performance loss in Fig. 9 compared with the AWGN channel. The BER curve with peak normalization in Fig. 10 does not go below the AWGN curve, although when using average normalization, it may happen. Thus, to avoid confusion in energy-limited adaptive transmission systems, we must use peak normalization of the channel, the expurgated average transmitted SNR referred to the receiver, and the expurgated BER.

\section{CONCLUSION}

Reliable and fair comparison of the performance of different systems that operate with different antenna configurations can be problematic. Both the average transmitted and received energies are used in performance comparisons, and the selection depends on what one wants to investigate. The transmitted energy is known to be the basic system resource. To avoid confusion in performance comparisons in energy-limited adaptive transmission systems, we must use peak normalization of the channel, the expurgated average transmitted SNR referred to the receiver, and the expurgated BER.

The whole idea of this paper is a generalization of the fact that, for correlated random variables $X$ and $Y$ and for $Z=$ $X Y$, we have the property $\mathbb{E}[Z]=\mathbb{E}[X Y]=\mathbb{E}[X] \mathbb{E}[Y]+$ $\operatorname{Cov}[X, Y]$. In our system model, $X$ corresponds to the transmitted energy, $Y$ corresponds to the energy gain of the channel, and $Z$ corresponds to the received energy. The random variables $X$ and $Y$ are correlated because of adaptive transmission, for example, when using transmitter power control. Therefore, the channel not only scales the transmitted energy, but the covariance $\operatorname{Cov}[X, Y]$ also plays a crucial role. The covariance describes how well the transmitted energy is statistically matched to the channel. If there are several orthogonal subchannels, a second covariance is needed to show how well the transmitted energy is statistically matched to the energy gains of the subchannels. In addition, the expression $\mathbb{E}[X] \mathbb{E}[Y]$ should not be referred to as the average received energy because the latter has the form $\mathbb{E}[X Y]$.

The systems under study should also be properly normalized. For example, it is reasonable to assume that a receiver equipped with two receiver antennas is able to receive twice as much energy as one with only one receiver antenna. The peak energy gains of the respective channel models should be scaled accordingly. Furthermore, the peak energy gain of the better channel should not exceed unity, or at the very least, the probability that the peak energy gain exceeds a respective limit should be the same for both channels.

\section{ACKNOWLEDGMENT}

The authors would like to thank I. Saarinen for useful discussions. The idea presented in this paper was a side product of his doctoral dissertation. 


\section{REFERENCES}

[1] M. P. Ristenbatt, "Alternatives in digital communications," Proc. IEEE, vol. 61, no. 6, pp. 703-721, Jun. 1973.

[2] R. W. Lucky, J. Salz, and E. J. Weldon, Principles of Data Communication. New York: McGraw-Hill, 1968.

[3] J. F. Hayes, "Adaptive feedback communications," IEEE Trans. Commun. Technol., vol. CT-16, no. 1, pp. 29-34, Feb. 1968.

[4] J. K. Cavers, "Variable-rate transmission for Rayleigh fading channels," IEEE Trans. Commun., vol. COM-20, no. 1, pp. 15-22, Feb. 1972.

[5] V. O. Hentinen, "Error performance for adaptive transmission on fading channels," IEEE Trans. Commun., vol. COM-22, no. 9, pp. 1331-1337, Sep. 1974.

[6] R. Srinivasan, "Feedback communications over fading channels," IEEE Trans. Commun., vol. COM-29, no. 1, pp. 50-57, Jan. 1981.

[7] A. Chockalingam, P. Dietrich, L. B. Milstein, and R. R. Rao, "Performance of closed-loop power control in DS-CDMA cellular systems," IEEE Trans. Veh. Technol., vol. 47, no. 3, pp. 774-789, Aug. 1998.

[8] D. M. Ionescu and A. Boariu, "Predictive closed-loop power control for frequency-division duplex wireless systems," IEEE Commun. Lett., vol. 5, no. 6, pp. 248-250, Jun. 2001

[9] E. Biglieri, J. Proakis, and S. Shamai, "Fading channels: Informationtheoretic and communication aspects," IEEE Trans. Inf. Theory, vol. 44, no. 6, pp. 2619-2692, Oct. 1998.

[10] J. G. Proakis, Digital Communications, 4th ed. New York: McGrawHill, 2001

[11] W. Xiang and S. S. Pietrobon, "On the capacity and normalization of ISI channels," IEEE Trans. Inf. Theory, vol. 49, no. 9, pp. 2263-2268, Sep. 2003.

[12] A. Mämmelä, I. Saarinen, and D. P. Taylor, "Transmitted energy as a basic system resource," in Proc. GLOBECOM, St. Louis, MO, 2005, pp. 3456-3460.

[13] A. Mämmelä, M. Höyhtyä, and D. P. Taylor, "Normalization of a fading channel," in Proc. GLOBECOM, San Francisco, CA, 2006, pp. 4254-4259.

[14] A. Kotelba, A. Mämmelä, and D. P. Taylor, "Normalization of linear vector channels," in Proc. GLOBECOM, Washington, DC, 2007, pp. 4537-4542.

[15] G. Raleigh and J. Cioffi, "Spatio-temporal coding for wireless communication," IEEE Trans. Commun., vol. 46, no. 3, pp. 357-366, Mar. 1998.

[16] S. K. Jayaweera and H. V. Poor, "Capacity of multiple-antenna systems with both receiver and transmitter channel state information," IEEE Trans. Inf. Theory, vol. 49, no. 10, pp. 2697-2709, Oct. 2003.

[17] A. M. Tulino and S. Verdú, "Random matrix theory and wireless communications," Found. Trends Commun. Inf. Theory, vol. 1, no. 1, pp. 1-182, Jun. 2004.

[18] E. Biglieri and G. Taricco, "Transmission and reception with multiple antennas: Theoretical foundations," Found. Trends Commun. Inf. Theory, vol. 1, no. 2, pp. 183-232, Oct. 2004.

[19] P. Lancaster and M. Tismenetsky, Theory of Matrices, 2nd ed. San Diego, CA: Academic, 1985.

[20] G. Caire and K. R. Kumar, "Information theoretic foundations of adaptive coded modulation," Proc. IEEE, vol. 95, no. 12, pp. 2274-2298, Dec. 2007.

[21] A. Staglione, P. Stoica, S. Barbarossa, G. B. Giannakis, and H. Sampath, "Optimal designs for space-time linear precoders and decoders," IEEE Trans. Signal Process., vol. 50, no. 5, pp. 1051-1064, May 2002.

[22] I. E. Telatar, "Capacity of multi-antenna Gaussian channels," Eur. Trans. Telecommun., vol. 10, no. 6, pp. 585-595, Nov. 1999.

[23] A. Lozano, A. Tulino, and S. Verdú, "Optimum power allocation for parallel Gaussian channels with arbitrary input distributions," IEEE Trans. Inf. Theory, vol. 52, no. 7, pp. 3033-3051, Jul. 2006.

[24] G. Caire, E. Biglieri, and G. Taricco, "Optimum power control over fading channels," IEEE Trans. Inf. Theory, vol. 45, no. 5, pp. 1468-1489, Jul. 1999.

[25] D. Gerlach and A. Paulraj, "Adaptive transmitting antenna arrays with feedback," IEEE Signal Process. Lett., vol. 1, no. 10, pp. 150-152, Oct. 1994

[26] U. Haagerup and S. Thorbjørnsen, "Random matrices with complex Gaussian entries," Expo. Math., vol. 21, no. 4, pp. 293-337, 2003.

[27] G. C. Clark and J. B. Cain, Error-Correction Coding for Digital Communications. New York: Plenum, 1981.

[28] T. Ratnarajah, R. Vaillancourt, and M. Alvo, "Complex random matrices and Rayleigh channel capacity," Commun. Inf. Syst., vol. 3, no. 2, pp. 119138, Oct. 2003.

[29] R. J. Muirhead, Aspects of Multivariate Statistical Theory. New York: Wiley, 1982
[30] A. T. James, "Distributions of matrix variates and latent roots derived from normal samples," Ann. Math. Stat., vol. 35, no. 2, pp. 475-501, Jun. 1964

[31] P. Koev and A. Edelman, "The efficient evaluation of the hypergeometric function of a matrix argument," Math. Comput., vol. 75, no. 254, pp. 833846, Jan. 2006

[32] M. Simon, "On the probability density function of the squared envelope of a sum of random phase vectors," IEEE Trans. Commun., vol. COM-33, no. 9, pp. 993-996, Sep. 1985.

[33] A. Abdi, H. Hashemi, and S. Nader-Esfahani, "On the PDF of the sum of random vectors," IEEE Trans. Commun., vol. 48, no. 1, pp. 7-12, Jan. 2000.

[34] G. D. Durgin, T. S. Rappaport, and D. A. de Wolf, "New analytical models and probability density functions for fading in wireless communications," IEEE Trans. Commun., vol. 50, no. 6, pp. 1005-1015, Jun. 2002.

[35] R. M. Gray, "Toeplitz and circulant matrices: A review," Found. Trends Commun. Inf. Theory, vol. 2, no. 3, pp. 155-239, 2006.

[36] A. Scaglione, "Statistical analysis of the capacity of MIMO frequency selective Rayleigh fading channels with arbitrary number of inputs and outputs," in Proc. ISIT, Lausanne, Switzerland, 2002, p. 278.

[37] B. Smith, "Six sigma design," IEEE Spectr, vol. 30, no. 9, pp. 43-47, Sep. 1993.

[38] M.-S. Alouini and A. J. Goldsmith, "Capacity of Rayleigh fading channels under different adaptive transmission and diversity-combining techniques," IEEE Trans. Veh. Technol., vol. 48, no. 4, pp. 1165-1181, Jul. 1999.

[39] A. Papoulis, Probability, Random Variables, and Stochastic Processes, 2nd ed. New York: McGraw-Hill, 1984.

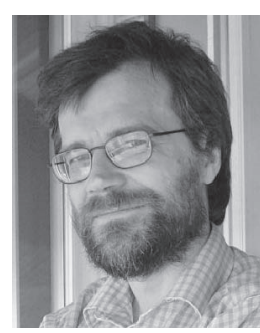

Aarne Mämmelä (M'83-SM'99) was born in Vihanti, Finland, in 1957. He received the M.Sc. (Tech.), Lic.Sc. (Tech.), and D.Sc. (Tech.) degrees (all with honors) from the University of Oulu, Oulu, Finland, in 1983, 1988, and 1996, respectively, all in electrical engineering. His main field of study was telecommunications.

From 1982 to 1993, he was an Assistant, Research Scientist, acting Associate Professor, and acting Professor with the Telecommunication Laboratory, University of Oulu, where he carried out research on adaptive algorithms in spread-spectrum systems. From 1990 to 1991 , he was a Visiting Research Scientist with the University of Kaiserslautern, Kaiserslautern, Germany. In 1993, he joined VTT Technical Research Centre of Finland, Oulu, as a Senior Research Scientist. He has been a Research Professor of digital signal processing in wireless communications since 1996. From 1996 to 1997, he was a Postdoctoral Research Scientist with the University of Canterbury, Christchurch, New Zealand. He has been an Adjunct Professor with the Helsinki University of Technology, Espoo, Finland, since 2000 and with the University of Oulu since 2004. Since 2001, he has regularly organized a course and given some of the lectures on research methodology for doctoral students in engineering at the University of Oulu. His research interests are in adaptive and cognitive systems in telecommunications.

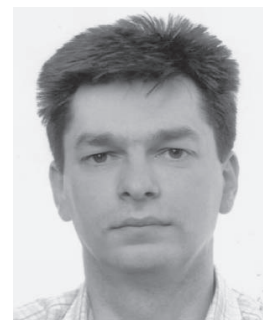

Adrian Kotelba (M'08) received the M.Sc. degree in electrical engineering from Poznan University of Technology, Poznan, Poland, in 2001. He is currently working toward the Ph.D. degree with the University of Oulu, Oulu, Finland.

He has been with VTT Technical Research Centre of Finland, Oulu, since 2000. His personal interests include communications theory, adaptive signal processing, adaptive modulation and coding, and cross-layer design. 


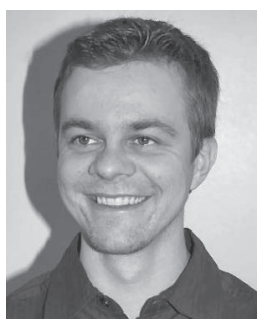

Marko Höyhtyä (S'07) received the M.Sc. degree in information engineering in 2005 from the University of Oulu, Oulu, Finland, where he is currently working toward the Ph.D. degree. His M.Sc. thesis was on adaptive transmitter power control in multipath fading channels.

Since the beginning of 2005 , he has been with VTT Technical Research Centre of Finland, Oulu, where he has been working on power control, beamforming, and cognitive radio technologies. Between February 2007 and February 2008, he made a oneyear research visit to the Berkeley Wireless Research Center, Berkeley, CA. His research interests include adaptive algorithms, transmitter power control, and spectrally efficient communication. He is particularly interested in adaptive power and frequency-allocation strategies in a cognitive radio network.

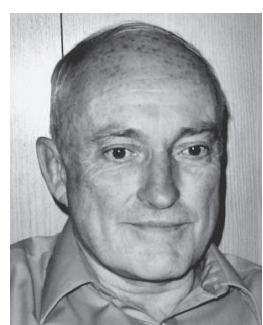

Desmond P. Taylor (M'65-SM'90-F'94-LF'07) received the Ph.D. degree in electrical engineering from McMaster University, Hamilton, ON, Canada, in 1972.

From July 1972 to June 1992, he was with the Communications Research Laboratory and Department of Electrical Engineering, McMaster University. Since 1992, he has been with the University of Canterbury, Christchurch, New Zealand, where he is currently the Tait Professor of Communications. $\mathrm{He}$ is the author or coauthor of approximately 230 published papers. He is the holder of two U.S. patents in spread-spectrum communications. His research is centered on digital wireless communications systems, with a primary focus on robust bandwidth-efficient modulation and coding techniques, and the development of iterative algorithms for joint equalization and decoding of fading dispersive channels. His secondary interests include problems in synchronization, multiple access, and networking.

Dr. Taylor is a Fellow of the Royal Society of New Zealand, the Engineering Institute of Canada, and the Institute of Professional Engineers of New Zealand. 